IJAMSR 2 (2) www.ijamsr.com CrossRef: https://doi.org/10.31426/ijamsr.2019.2.2.1215

International Journal of

I J A M S R

Advanced Multidisciplinary Scientific Research (IJAMSR) ISSN:2581-4281

\title{
BIOLOGICAL PHENOMENA MIMIC ASTROPHYSICAL PRINCIPLES: UNIFIED PERSPECTIVE ON HUMAN ACCRETION MASS, MUSCLE TONE TENSION AND GLIMMERING BIOLUMINESCENCE
}

\author{
Dr. Iresh Ranjan Bhattacharjee, \\ Principle Scientist, Institute for Intrinsic Gravitation Biology \\ Email: selfgravity@gmail.com
}

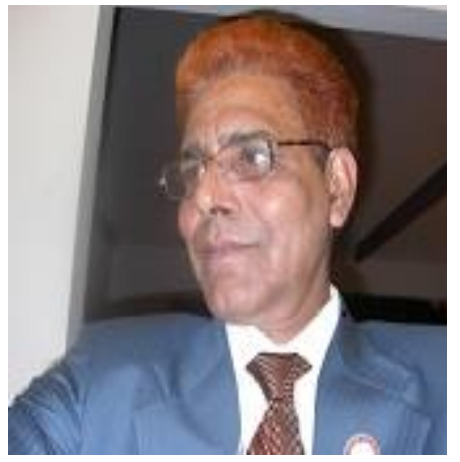

DR.IRESH RANJAN BATTACHARJEE

Keywords: Accretion mass, accumulation of particles, galaxies, stars, and planets

\begin{abstract}
A B S T RA C T
Accretion mass is a term used in astrophysics to signify accumulation of particles that are gravitationally more attractive than non-accumulated mass, such as gases or fluids. Accretion is the gradual increase in size by the build up of matter due to gravity. Most astronomical objects, such as galaxies, stars, and planets, are formed through accretion processes from gaseous state. The term accretion mass has not yet incorporated in the life science to describe many biological situations like the difference between old and newborn, differential anatomical features or sporting standard for men and women, etc. Muscle tone, on the other hand, is regarded as an inherent elasticity in the muscles. The intrinsic muscle tone is constantly maintained when living but said to lose after death. The reason for the phenomena is not yet explained in health science. Lastly, the spontaneous origins and mechanisms of ultraweak photon (light) emission from living systems are not yet well understood. These are analyzed with a unified view that biological phenomena mimic astrophysical principles. Inward gravitational force is counterbalanced by outward pressure or metabolic energy to be considered as living. In all these three discrete events in life science, such phenomena do exist. Astrophysical principle states that under hydrostatic equilibrium, gravitational compressive energy is compensated by outward radiant energy. Gravitation and metabolic energy work in tandem in the diagonally opposite manner under an equilibrium state from zygote-foetus stages till death with shining in respective waves during life time. Shining or tuning dies down with death under non-equilibrium state.
\end{abstract}

Citation: Iresh Ranjan Bhattacharjee, (2019). Biological Phenomena Mimic Astrophysical Principles: Unified Perspective On Human Accretion Mass, Muscle Tone Tension And Glimmering Bioluminescence. International Journal of Advanced Multidisciplinary Scientific Research (IJAMSR ) ISSN:2581-4281, 2 (2), February, 2019, \# Art.1215, pp 55-67 
IJAMSR 2 (2) www.ijamsr.com CrossRef: https://doi.org/10.31426/ijamsr.2019.2.2.1215

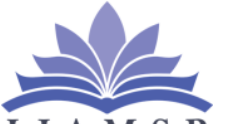

\section{International Journal of \\ Advanced Multidisciplinary Scientific Research (IJAMSR) ISSN:2581-4281}

I J A M S R

\section{Introduction}

Accretion mass is a term used in astrophysics to signify accumulation of particles that are gravitationally more attractive than nonaccumulated mass, such as gases (or in case of biology, fluids). Accretion is the gradual increase in size by the build-up of matter due to gravity. Most astronomical objects, such as galaxies, stars, and planets, are formed through accretion processes from gaseous state. The term accretion mass has not yet incorporated in the life science to describe many biological situations like the difference between old and newborn, differential sporting standard for men and women, etc. Muscle tone, on the other hand, is regarded as an inherent elasticity in the muscles. The intrinsic muscle tone is constantly maintained when living but said to lose after death. The reason for the phenomena is not yet explained in health science. Lastly, the spontaneous origins and mechanisms of ultraweak photon (light) emission from living systems are not yet well understood. The present study was therefore made to find out whether a unified code for these three discrete phenomena in life science could be worked out or not in conformity with the astrophysical principles.

\section{Accretion Mass}

In our previous articles ${ }^{1-4}$, we have projected that fluids being gravitationally less active than accreted granular materials work as astronomical distance. In homogenous accretion, similar elements stick together and heavier particles sink towards centre of mass. In heterogeneous accretion, heavier elements collects in the centre and lighter particles adhere to the mass (Illustration 1). Accretion mass is being coined from astrophysics. It is deemed to fit well to describe various biological phenomena.

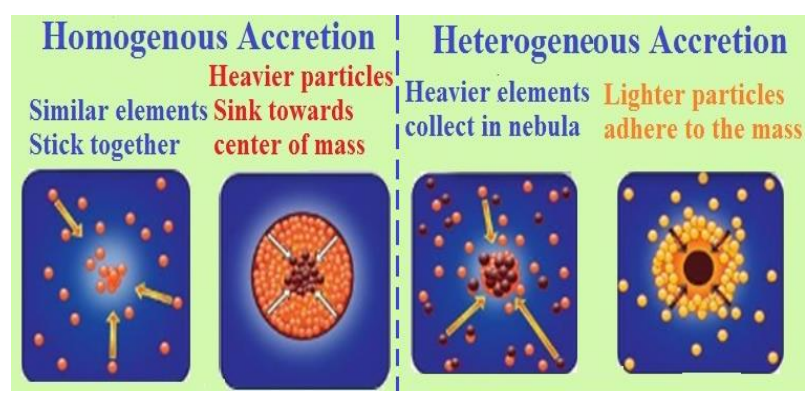

Illustration 1: In homogenous accretion, similar elements stick together and heavier particles sink towards centre of mass. In heterogeneous accretion, heavier elements collects in the centre and lighter particles adhere to the mass.

Accretion mass is considered matters that are solid, in semi-solid granular or in soft condensed matter phase including interstitial one and not fluids. In a body of an elderly person, created materials are more than that of newborn. 
IJAMSR 2 (2) www.ijamsr.com CrossRef: https://doi.org/10.31426/ijamsr.2019.2.2.1215

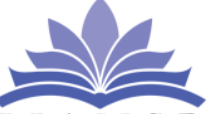

\section{International Journal of Advanced Multidisciplinary Scientific Research (IJAMSR) ISSN:2581-4281}

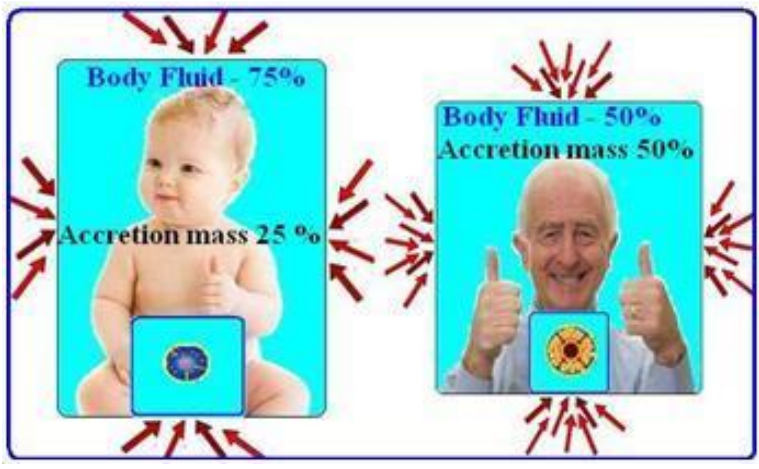

Illustration 2: Difference in accretion mass and body fluids in child and old.

It is about $50 \%$ in the elderly; where as new born baby is over about $25 \%$. New born is, however, composed of more water $(75 \%)$ than that of an elderly person (50\%) (Illustration 2).

The reason behind the introduction of accretion mass is to differentiate between buoyant and gravitational mechanism, due to their individual physical property in a gravitating environment. The fluids, like gases, are more an indicator of the buoyant property than gravitational property. Thus, elderly persons are more prone to higher gravitational attraction. The athletic spirit- running, jumping and so on of the elderly persons goes on decreasing with age with accumulation of accretion mass, no matter what metabolic energy gets supplemented.

\section{Difference in anatomical and sporting} standards between Men and Women.

We have earlier ${ }^{1}$ pointed out that under neutral buoyancy, different class of macromolecules tends to occupy positions within a biological cell as per their molar mass and density due to action of self gravity (Illustration 3).

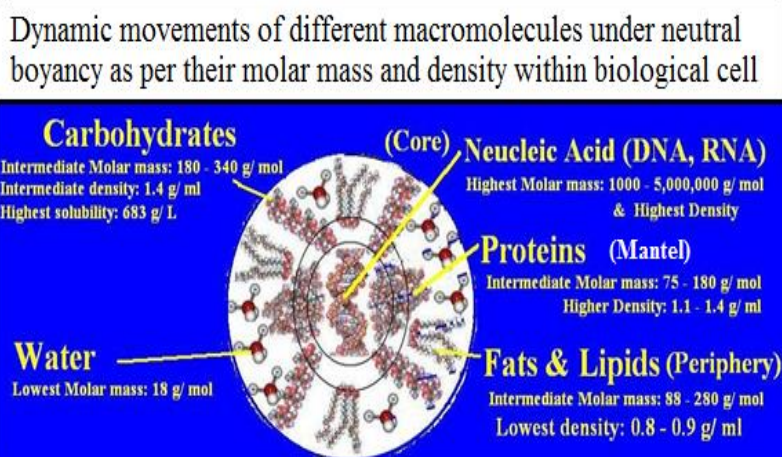

Illustration 3: Macromolecules having higher molar mass and density tend to occupy a central position.

Heavier macromolecules like nucleic acid having a molar mass of 1,000,000 to 5,000,000 $\mathrm{g} /$ mole under tightly packed condition (highest density) tend to occupy the core position of the three dimensional biological cell, similar to collection of heterogeneous heavier elements in the nebula in the astrophysical objects. Proteins, being intermediate molar mass of 75$180 \mathrm{~g} / \mathrm{mol}$ tend to occupy mantel position; fats 
IJAMSR 2 (2) www.ijamsr.com CrossRef: https://doi.org/10.31426/ijamsr.2019.2.2.1215

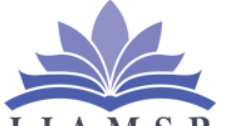

\section{International Journal of \\ Advanced Multidisciplinary Scientific Research (IJAMSR) ISSN:2581-4281}

and lipids in the periphery due to their lowest density (0.8 -0.9 g/ ml). Carbohydrates evade central attraction due to their higher miscibility. The protoplasm is granular colloidal in nature, because many macromolecules, tiny organelles are suspended in it, also exhibits sol and gel properties. Gary Michael Gutt $^{5}$ while studying physics of granular systems in 1989 at California Institute of Technology expressed that granular system exhibit duel properties - in a gravitational field, it may have a self-bounding free surface, but also conform to the shape of the bounding wall, obeying continuum theories of fluid mechanics. As a compacted granular system, it can support shear stress in the absence of a shearing velocity. Application of granular physics is still at a nascent stage of study in life science. There is need for in-depth study on application of granular physics in life science to advance further on carbohydrate and other macromolecular movements.

In this matter, let us study why anatomical intrusion (ovary) and extrusion (testes) characteristics occurs or sporting standards between women and men differs in the light of aforesaid gravitational environment. The average human body is said to contain approximately 37.2 trillion $\left(3.72 \times 10^{13}\right)$ cells.
Within the nucleus of every cell, there is a genetic code, say DNA that remains under tightly packed macromolecular condition. Out of 46 (22 pairs) chromosomes in human, the genetic differences between men and women lie in sex chromosomes. In the sex chromosomes or 23rd pair, females have two copies of the $\mathrm{X}$ chromosome, while males have one $\mathrm{X}$ and one $\mathrm{Y}$ chromosome. $\mathrm{Y}$ chromosomes are about one-third the size of $X$ chromosomes. The $\mathrm{X}$ chromosome represents about 5 percent of the total DNA in cells, while the $\mathrm{Y}$ chromosome represents about 2 percent of a cell's total DNA. Molar mass weight is therefore higher in women than men.

\section{Female $\mathrm{X}$ chromosome has higher molar mass than male XY}

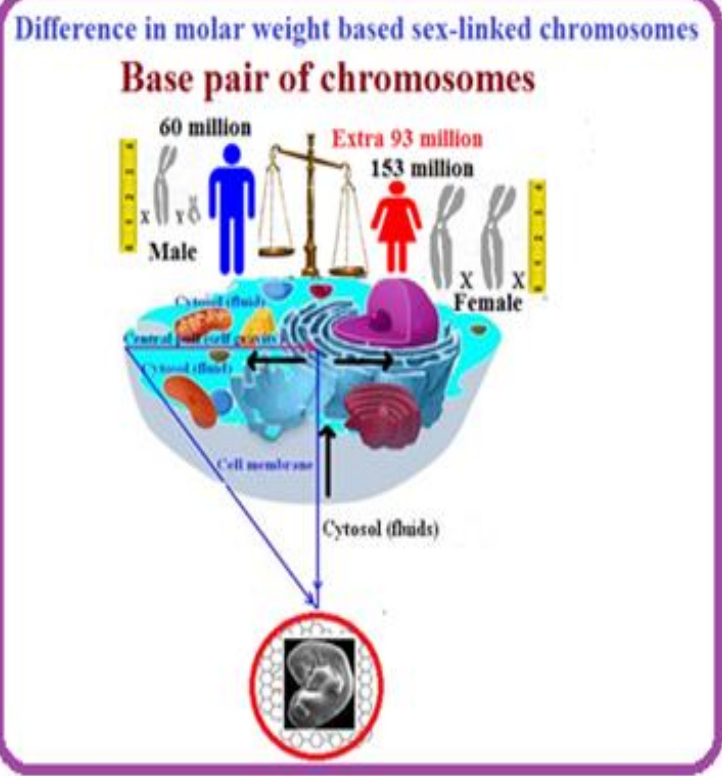


IJAMSR 2 (2) www.ijamsr.com CrossRef: https://doi.org/10.31426/ijamsr.2019.2.2.1215

\section{International Journal of Advanced Multidisciplinary Scientific Research (IJAMSR) ISSN:2581-4281}

Illustration 4. Pictorial demonstration 1. There is comparatively higher weighty accretion mass in females than males. Females contain 153 million compared to 60 million base pairs of chromosomes for men. Females contain extra 93 million base pairs of chromosomes.

Molecular weight or molar mass of single strand DNA $=650 \mathrm{~g} / \mathrm{mol} . \quad\left(6.022 \times 10^{23}=\right.$ mole or Avogadro's number). One mole of a base pair weighs $650 \mathrm{~g}$. The molecular weight or molar mass of any double stranded DNA fragment can therefore be calculated by multiplying its length (in bp) by 650 and the answer will be expressed as $\mathrm{g} / \mathrm{mol}$ or daltons. It is to be noted that chromosome $\mathrm{X}$ has 153 million $\left(1,000,000\right.$ or $\left.10^{6}\right)$ base pairs. On the other hand, chromosome $\mathrm{Y}$ has 60 million base pairs. Thus females having chromosome $\mathrm{X}$ contain extra 93 million base pairs. The molecular weight of accretion masses in females are thus higher (> one and a half times) than their counterpart males (Illustration 4). In the standard course of events, dense heavier macromolecules dipped in granular soft viscoelastic condensed matter, the central traction force from the dynamic core of the embryonic mass leads to either testes or embryo, depending on the comparative availability of higher or lower quantity of chromosomes in terms of molar mass and density in the nucleic acid materials. An embryo with two $\mathrm{X}$ chromosomes, having highest molar mass, becomes a girl, while an embryo with an $\mathrm{X}$ and a $\mathrm{Y}$ chromosome, having less molar mass, becomes a boy.

We have earlier ${ }^{3}$ pointed out that 'mass' has a dual role. One is a synonym of 'gravitation' and other is 'metabolic energy'. The compression energy of self gravity in living mass is required to be counterbalanced by noncompressive metabolic energy (ME) to remain an organism as "living". Basal metabolic rate (BMR) i.e. consumption of oxygen molecule per hour is $2 / 3$ or $3 / 4$ power of mass. Naturally, when gravitational energy gets higher with higher molar weight per unit in females, the efficiency of metabolic energy would be less. This is the reason why general sporting standards differ between men and women athletes, women's standard being less than men or, men are typically taller than women. The distinction between male and female in height and sex is therefore proposed to be due to difference in molar weight of sex-linked chromosomes under maximum gravitation compression at the contemporary core in viscoelastic soft bio matters of the embryo. Generally males are taller than female with 
IJAMSR 2 (2) www.ijamsr.com CrossRef: https://doi.org/10.31426/ijamsr.2019.2.2.1215

\section{International Journal of Advanced Multidisciplinary Scientific Research (IJAMSR) ISSN:2581-4281}

extrusion sex (testes) feature, whereas females are, on average shorter in height with intrusion type (ovary) sex feature. This is deemed to be happening due to difference in molar weight based sex-linked chromosomes, because in case of women, due to extra 93 millions of base pairs of the chromosomes in each cell, the soft condensed mass of hypothetical 37.2 trillion $\left(3.72 \times 10^{13}\right)$ cells would experience higher inward gravitational pressure, as pictorially demonstrated in (Illustration 5).

Distinction between male and female in height and sex Difference due to molar weight of sex-linked chromosomes

$\begin{gathered}\text { Gravitation compression in viscoeleastic } \\ \text { soft biomatters in embryo }\end{gathered}$
Sex chromosome
Intrusion

Illustration 5. Pictorial demonstration 2. The distinction between male and female in height and sex is proposed to be due to difference in molar weight of sex-linked chromosomes under maximum gravitation compression at the contemporary core in viscoelastic soft bio matters of the embryo. Generally males are taller than female with extrusion sex (testes) feature, whereas females are, on average shorter in height with intrusion type (ovary) sex feature.

\section{Muscle Tone}

Muscle tone ${ }^{6}$ is usually defined as the amount of contraction in a resting muscle. It is a static, balanced, isometric contraction between agonist and antagonist (both internal forces) in every muscle in the body. It never lets go completely, when one does nothing. Even in sleep, when all dynamic muscle activity is gone, the tone remains. Muscle-building exercise elevates tone, can even slam it up considerably, but at whatever level the involvement of contraction, tone still remains static and constant at any given time. In our earlier publications, we have pointed out that sudden jerking or convulsive movement of many fibers, excited asynchronously at low frequencies up to $5 /$ second with amplitude is proportional to the average frequency of excitation. Summation of the twitches of many fibers produces 'background' tension. All the muscles in a living organism possess such tone or background tension.

Even in a relaxed limb, the motor nerves are activated at low frequency. However, an electromyograph can’t pick up such low level, steady-state action unless the motor units immediately adjacent to the contact are firing. It sometimes read as background noise. Also, it is regarded occasionally as due to inherent 
IJAMSR 2 (2) www.ijamsr.com CrossRef: https://doi.org/10.31426/ijamsr.2019.2.2.1215

\section{International Journal of Advanced Multidisciplinary Scientific Research (IJAMSR) ISSN:2581-4281}

elasticity in the muscles. The most interesting fact is that intrinsic muscle tone is constantly maintained when living but loose after death.

Generally the tone is said to be 'regulated' (decreased or increased) by virtue of sympathetic and parasympathetic supply. The autonomic nervous system (ANS) operates without conscious control, relying upon reflex arcs that are dependent upon hypothalamus and medulla for overriding control. Autonomic nerve fibers innervate cardiac muscle, smooth muscle, and glands. Through these fibers the ANS said to play a role in regulating blood pressure and flow, gastrointestinal movements and secretions, body temperature, bronchial dilation, blood glucose levels, metabolism, micturition and defecation, pupillary light and accommodation reflexes, and glandular secretions, just to name a few.

Paralysis is the loss or impairment of motor function in a part due to a lesion of the neural or muscular mechanism. Paralyzed muscle may be flaccid, flabby, and without appreciable tone, or it may be spastic, tight, and with an abnormally high tone that increases when the muscle is moved. But the most interesting fact is that there is no complete loss of background tone during paralysis. Paralysis is most often caused by damage in the nervous system, especially the spinal cord. It takes about onethousandth of a second for a neuron to fire an impulse and return to its resting level. Both sympathetic and parasympathetic neurons have a constant rate of firing under normal conditions. This is also called their "tone", but of neural origin. It maintains a normal rate of heartbeat, keeps blood pressure within a normal range and maintains homeostasis. Of course, the sympathetic and parasympathetic firing rate changes greatly during fight-orflight responses.

Complete loss of tone is only available with death. At the moment of death, all of the muscles in the body relax, a state called primary flaccidity. Eyelids lose their tension, the pupils dilate, the jaw might fall open, and the body's joints and limbs are flexible. With the loss of tension in the muscles, the skin will sag, which can cause prominent joints and bones in the body, such as the jaw or hips, to become pronounced. During the process called a pallor mortise (within minutes of the heart stopping) blood drains from the smaller veins in the skin and the original background tone is lost. Nerve impulse fails to act when clinically dead. During the process called livor mortis (2 to 6 hours after death), hypostasis or settling of blood occurs. Fluids accumulate in lower or 
IJAMSR 2 (2) www.ijamsr.com CrossRef: https://doi.org/10.31426/ijamsr.2019.2.2.1215

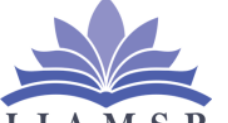

International Journal of

Advanced Multidisciplinary Scientific Research (IJAMSR) ISSN:2581-4281

dependent parts of an organ or body (dependent edema). Blood come out to the areas closest to the ground or external gravitational field. The organism behaves as inert on losing its tone (Illustration 6). Why downward pull towards external direction? Why such severe manifestation of earth's gravity is not pronounced while living? The length of the small intestine remains at half while living due to the tone in the longitudinal muscle bundles in the gut. After a death gut length becomes double, say about $24 \mathrm{ft}$. What is hidden source of intrinsic tension? Thus we can perceive that full tone is due to balance between the potential energy of self gravity and kinetic energy of metabolism. The tone is lost and organism behaves as inert matter out of imbalance between inner potential and kinetic energies when stronger external gravity dominates.

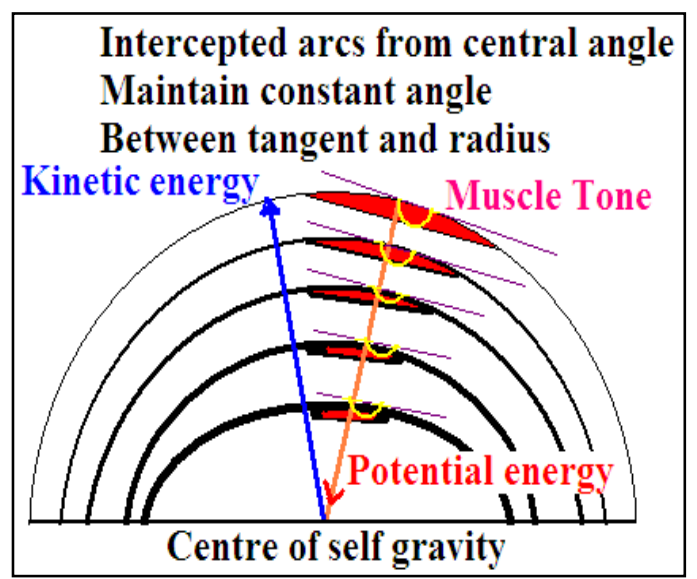

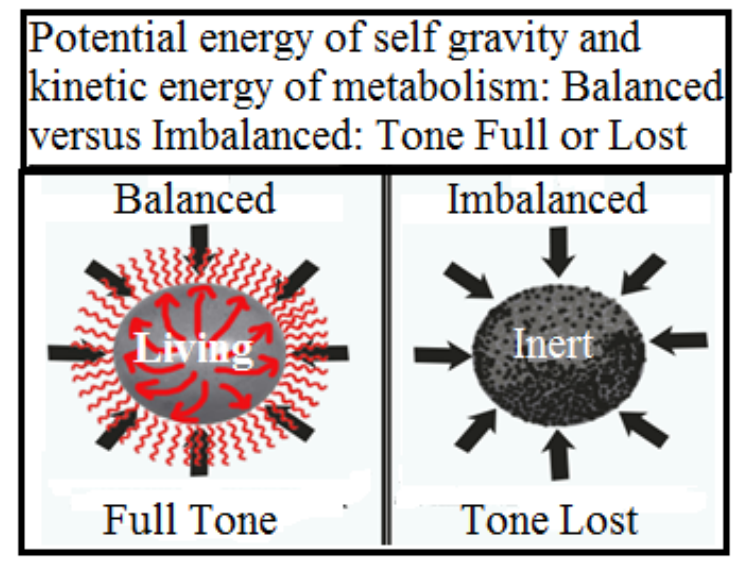

Illustration 6. Schematic diagram shows, full tone due to balance between the potential energy of self gravity and kinetic energy of metabolism (left). The tone is lost and organism behaves as inert matter out of imbalance between inner potential and kinetic energies when stronger external gravity dominates (right).

\section{Ultra weak photon (light) emission}

Like any astrophysical luminary bodies, researchers had observed universal phenomenon of all living systems on glimmering bioluminescence through ultra weak photon (light) emission. However, these biophotons ranges in intensity from a few to approximately $10^{2}$ photons / $\left(\mathrm{s} \times \mathrm{cm}^{2}\right)$. It is thus not visible to the naked eye and cannot be captured with commonly used optical detectors. The spectral range is 400-720 nm. Masaki Kobayashi et al ${ }^{7}$, the biomedical 
IJAMSR 2 (2) www.ijamsr.com CrossRef: https://doi.org/10.31426/ijamsr.2019.2.2.1215

\section{International Journal of Advanced Multidisciplinary Scientific Research (IJAMSR) ISSN:2581-4281}

Photonics team at the Tohoku Institute of Technology in Sendai, Japan using supersensitive cameras monitored five healthy male volunteers for 20 minutes every 3 hours inside a light-tight room for three days and found that participants 'glowed' throughout the day, with the bright spots appearing around the forehead, neck, cheeks in the late afternoon. The dimmest bioluminescence was recorded late at night (Illustration 7).

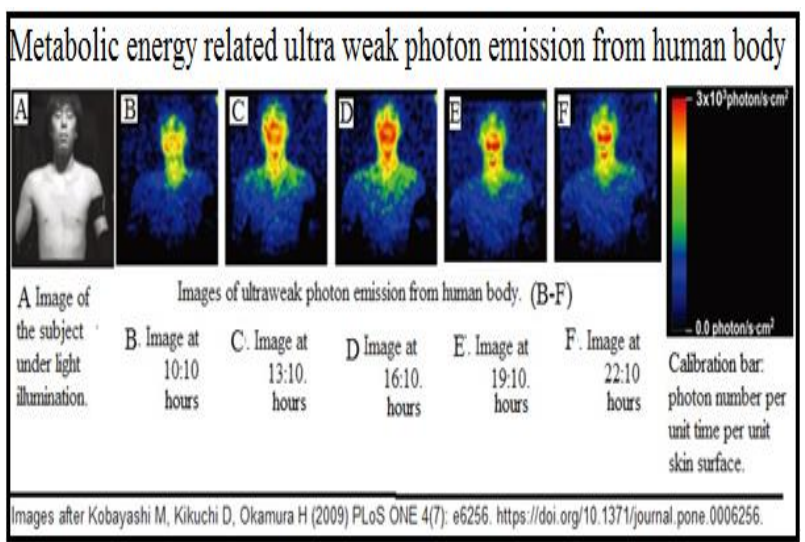

Illustration 7 Images of ultra weak photon emission (photon number per unit time per unit skin surface) from healthy human body are presented after Kobayashi et al (2009). Photon emission was lowest in the morning at 10:10 hours (Image B) and highest at few hours after meal at 6-10 hours (Image D). Glimmering effects decreases subsequently at 19:10 hours (Image E) and 22:10 hours (Image F).
These were not infrared radiation caused by heat but a side-effect of metabolisms. They were of the opinion that the diurnal changes in photon emission might be linked to changes in energy metabolism. There is less emission with patients suffering from disease like hypothyroidism where lower state of metabolic activity than normal is encountered. Photon counting statistics on the distribution of photons in a biophoton signal was also studied. Higher probability moments of biophotons from healthy subjects were noticed corresponding to Poisson distribution while those of paralytic persons revealed deviations from Poisson distribution and seemed to approach geometrical distribution. Geometrical distribution to paralytic persons is explained in terms of the interrelations of the underlying metabolic processes. Thus, detection of emission could provide a new non-invasive and powerful tool for investigating cellular tissues apart from health, medical diagnostic tool in terms of basic regulatory functions of the body including body field.

Group elsewhere8 have investigated various influencing other factors like age and gender (higher emissions from the hands in case of elderly people) so far photon emissions are concerned. We have already pointed out that elderly person possesses higher gravitational 
IJAMSR 2 (2) www.ijamsr.com CrossRef: https://doi.org/10.31426/ijamsr.2019.2.2.1215

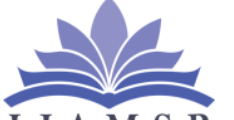

\section{International Journal of}

Advanced Multidisciplinary Scientific Research (IJAMSR) ISSN:2581-4281

relevant accredited mass than the younger ones. Moreover, there is left-right symmetry in glimmering bioluminescence within the human body which, we suppose, is intricately related to the 'convectional flow phenomena under self gravitating environment from the center of mass' (Illustration 8), as explained in our earlier publications. Light channels in the body are linked with disease in terms of broken symmetry between left and right side or regulation of energy and information transfer between different parts.

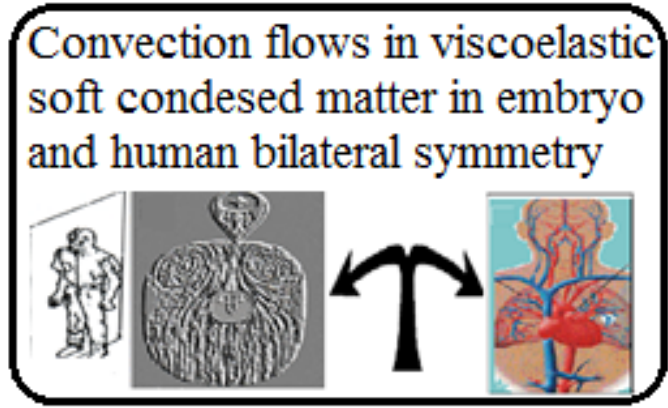

Illustration 8. Pictorial demonstration 3: Bilateral or mirror symmetry, temperature and pressure regulated convection flows during embryonic growth and developments.

The compressive energy of self gravitation would be available in a human circulating system for initiating from foetus stage. Heart starts functioning at 31 days of the embryonic stage, when it could be at the geometrical centre of the foetus floating in amniotic fluid.
The total gravitational attraction of the foetal mass gets reduced due to buoyant force. 'Planet within the planet situation creates. With further development and accumulation of mass, the geometrical center gets shifted from heart to somewhere in the lower part of the abdomen say below the umbilical cord (Illustration 10).

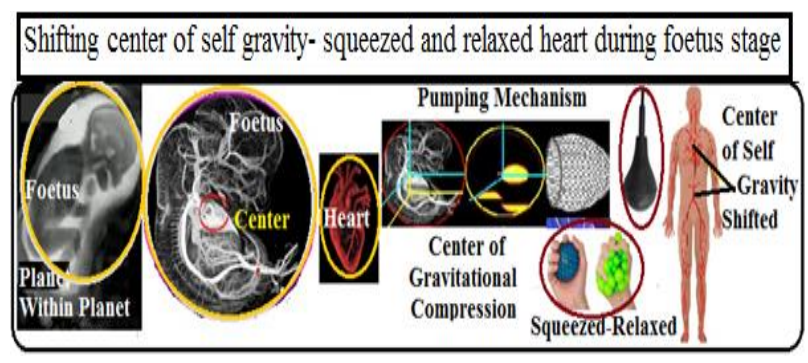

Illustration 9. Pictorial demonstration 4: Foetus under amniotic fluid is as if planet within planet situation, heart in the centre of 31 days embryo, it undergoes gravitational squeezing and relaxed pumping system; with further growth and development, center of self gravity gets shifted.

Inhalation and exhalation originate from the imaginary center of self gravity with curvature of diaphragm contracting and relaxing has been pictorially demonstrated in (Illustration 9). 
IJAMSR 2 (2) www.ijamsr.com CrossRef: https://doi.org/10.31426/ijamsr.2019.2.2.1215

\section{International Journal of}

I J A M S R

Advanced Multidisciplinary Scientific Research (IJAMSR) ISSN:2581-4281

\section{Contracting - Relaxing from centre of self gravity during respiration

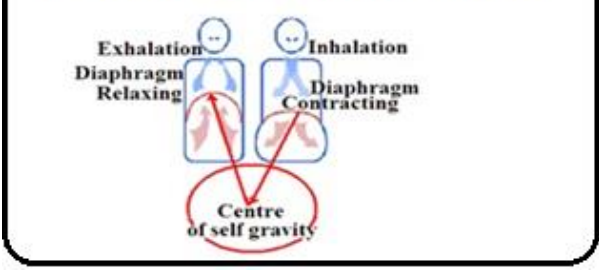

Illustration 10. Pictorial demonstration 5: Inhalation and exhalation originating from the imaginary center of self gravity with curvature of diaphragm contracting and relaxing.

In fact, blood flows through neatly woven channel networks. Blood is regarded as highly cooperative, non-equilibrium and non-linear system, whose components unceasingly interact in time and space in which blood has the property to store electron excitation that is produced in the course of its own normal metabolism (Illustration 11).

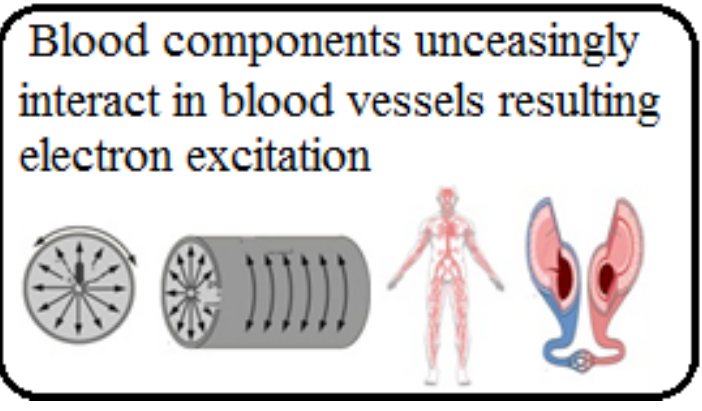

Illustration 11. Pictorial demonstration 6 . Blood components continuously interact so also store metabolically produced excited electron.
Voeikov et al (2003) ${ }^{9}$ reported that pumping out of blood is a continuous source of biophotons. Blood persists in an electronically excited state in the presence of reactive oxygen and neutrophil suspensions. These remain in an oscillatory state. Such excited state of blood is extremely sensitive to the tiniest fluctuations of external photonic fields, but resistant to temperature variations as reflected in hysteresis of photon emission in response to temperature variations.

\section{A unified perspective on human accretion} mass, muscle tone tension and glimmering

\section{bioluminescence}

In our earlier publication, we have shown the stages of formation of planets wherein homogenous low density protoplanet after initial accretion becomes dense and differentiated planet on being exposed to gravitational contraction, size reduction and increase in density. In the core, gravitational compression produces heat. Gravitational pressure is then balanced by the outward thermal pressure from fusion reactions, temporarily halting gravitational compression. Equilibrium is reached when the compression is balanced by the pressure gradient. This pressure gradient is in the opposite direction due to the strength of the material, at which 
IJAMSR 2 (2) www.ijamsr.com CrossRef: https://doi.org/10.31426/ijamsr.2019.2.2.1215

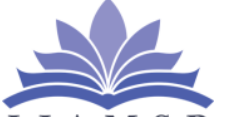

\section{International Journal of}

Advanced Multidisciplinary Scientific Research (IJAMSR) ISSN:2581-4281

point gravitational compression ceases. death under non-equilibrium state.

Biological body also shows the similar phenomena, but with a feeble magnitude. Say instead of heat, one may find a minor variation in temperature and so on.

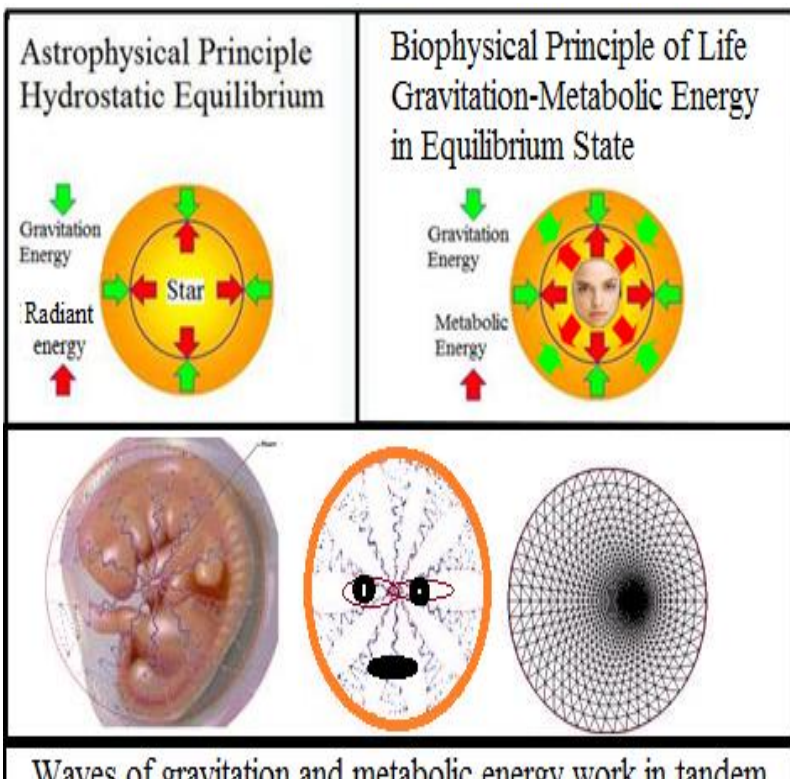

Waves of gravitation and metabolic energy work in tandem

Illustration 12. The waves of gravitation and metabolic energy work in tandem.

\section{Conclusion}

Astrophysical principle states that under hydrostatic equilibrium, gravitational compressive energy is counterbalanced by outward radiant energy. Gravitation and metabolic energy work in tendem in diagonially opposite manner under equilibrium state from zygote-foetus stages till death with shining in respective waves during life time. Shining die down with 
IJAMSR 2 (2) www.ijamsr.com CrossRef: https://doi.org/10.31426/ijamsr.2019.2.2.1215

\section{International Journal of \\ Advanced Multidisciplinary Scientific Research (IJAMSR) ISSN:2581-4281}

\section{References}

1) Iresh Ranjan Bhattacharjee (2018). Biological Phenomena Mimic Astrophysical Principles Of Gravitation - Opening Perspective. International Journal of Advanced Multidisciplinary Scientific Research (IJAMSR ) ISSN:2581-4281 Vol 1, Issue 6, August, 2018, \#Art.611, pp1-16. https://doi.org/10.31426/ijamsr.2018.1. 6.611

2) Iresh Ranjan Bhattacharjee (2017) Gravitation Induced Mechanical Movement in Cells. Journal for Research | Volume 03| Issue 01 | March 2017 ISSN: 2395-7549. http://www.journal4research.org/articl es/J4RV3I1015.pdf

3) Bhattacharjee, IR. (2013) Self Gravity: The Major Investigation Gap in Life Science (Part I). WebmedCentral BIOPHYSICS; 4(6):WMC004279

a. doi:10.9754/journal.wmc.2013. 004279

4) Bhattacharjee, IR. (2013) Self Gravity: The Major Investigation Gap in Life Science (Part II). WebmedCentral BIOPHYSICS;4(6):WMC004296

a. doi: 10.9754/journal.wmc.2013.0042 96

5) Gutt, Gary Michael. The physics of granular systems. Ph.D. Dissertation, 1989 California Institute of Technology. http://resolver.caltech.edu/CaltechETD :etd-05302007-081951

6) http://www.neurosoma.com/muscletone .html
7) Kobayashi M, Kikuchi D, Okamura $H$. Imaging of Ultraweak Spontaneous Photon Emission from Human Body Displaying Diurnal Rhythm. PLoS ONE (2009) 4(7): e6256. https://doi.org/10.1371/journal.pone.00 06256

8) https://www.researchgate.net/publicati on/8465511_Biophoton_research_in_bl ood_reveals_its_holistic_properties

9) Voeikov VL1, Asfaramov R, Bouravleva $E V$, Novikov CN, Vilenskaya ND. Biophoton research in blood reveals its holistic properties. Indian J Exp Biol. 2003 May;41(5):473-82. 